\title{
Geranylgeranylacetone Protects Guinea Pig Gastric Mucosal Cells from Gastric Stressor-Induced Necrosis by Induction of Heat-Shock Proteins
}

\author{
Wataru Tomisato, ${ }^{a}$ Shinji Tsutsumi,${ }^{a}$ Tomofusa Tsuchiya, ${ }^{a}$ and Tohru Mizushima ${ }^{*, a, b}$ \\ Faculty of Pharmaceutical Sciences, Okayama University ${ }^{a}$ and PRESTO, Japan Science and Technology Corporation, ${ }^{b}$ \\ 1-1-1 Tsushima-naka, Okayama 700-8530, Japan. Received March 9, 2001; accepted May 8, 2001
}

\begin{abstract}
Gastric mucosal cell death due to various gastric stressors can cause several types of gastric diseases, such as gastric ulcers. In this study, we examined cell death following the short-term treatment of guinea pig gastric mucosal cells in primary culture with various gastric stressors. The short-term treatment of cells with ethanol, hydrogen peroxide or hydrochloric acid caused, in a dose-dependent manner, cell death in the absence of apoptotic DNA fragmentation and chromatin condensation. Cells lost membrane integrity following the treatment with each of these gastric stressors, suggesting that necrosis was induced in gastric mucosal cells by short-term treatment of the cells with gastric stressors. Geranylgeranylacetone, an anti-ulcer drug with heat-shock protein inducing properties, protected gastric mucosal cells from the necrotic cell death caused by each of these gastric stressors. Pretreatment of cells with low concentrations of ethanol $(3 \%)$, which also induced heat-shock protein, made cells resistant to the necrotic cell death caused by the gastric stressors. These results suggest that heatshock proteins is involved in the cytoprotective effect of geranylgeranylacetone against necrotic cell death.
\end{abstract}

Key words geranylgeranylacetone; heat-shock protein; necrosis; cytoprotective effect; gastric mucosal cell; gastric stressor

The gastric mucosa in vivo is exposed to various types of stressors (such as alcohol, acid, oxidative stressor, drug, and bacteria) which can cause cell death resulting in gastric ulcers and gastritis. The protection of gastric mucosal cells from gastric stressor-induced cell death would therefore be therapeutically beneficial for preventing such stressor-induced gastric mucosal damage. However, the clinical means by which to do this are yet to be established, primarily because the mechanism governing gastric stressor-induced cell death is still unknown. As a first step to the elucidation of this process, gastric stressor-induced cell death must be reproduced in vitro. This can be done using gastric mucosal cells in primary culture whose properties closely mimic those of gastric mucosal cells in vivo.

Gastric mucosal cells are thought to be damaged through both necrosis and apoptosis in vivo. ${ }^{1-4)}$ We recently found that non-steroidal anti-inflammatory drugs (NSAIDs), one of the major gastric stressors, induce both necrosis and apoptosis depending on the treatment conditions; short-term treatment of cells with high concentrations of NSAIDs and longterm treatment of cells with low concentrations of NSAIDs induce necrosis and apoptosis, respectively, in guinea pig gastric mucosal cells in primary culture (Tomisato et al., submitted). In relation to gastric stressors other than NSAIDs, we also found that long-term treatment of cells with ethanol, hydrogen peroxide, and hydrochloric acid induced apoptosis in gastric mucosal cells in primary culture (Tsutsumi et al., submitted). Thus, it is reasonable to postulate that these gastric stressors induced necrosis when gastric mucosal cells were treated with these compounds for short periods.

When cells are exposed to various stressors, the expression of a distinct group of proteins, called heat shock proteins (HSPs), is induced which make cells resistant to various stressors. HSPs are generally thought to be important for gastric mucosal defense. ${ }^{5)}$ Therefore, it seems that pre-induction of HSPs in gastric mucosal cells protects the cells from gastric stressor-induced necrosis and would be of therapeutic benefit for preventing gastric mucosal damage. Supporting this notion, geranylgeranylacetone (GGA), an anti-ulcer drug, was shown to directly induce HSPs in cultured gastric mucosal cells and in intact gastric mucosa. ${ }^{6}$ This HSP-induction protect cells from ethanol-induced cell death. ${ }^{6)} \mathrm{We}$ also showed that pre-induction of HSPs by GGA prevents subsequent necrosis by NSAIDs in guinea pig gastric mucosal cells in primary culture. ${ }^{7)}$ Thus, it is reasonable to predict that pre-induction of HSPs by GGA also prevents subsequent necrosis by gastric stressors other than NSAIDs. We show here that short-term treatment with various gastric stressors (ethanol, hydrogen peroxide and hydrochloric acid) induces necrosis in guinea pig gastric mucosal cells in primary culture. We also found that pre-treatment of cells with GGA or low concentrations of ethanol, both of which induced expression of HSPs made cells resistant to necrotic cell death caused by each of the gastric stressors.

\section{MATERIALS AND METHODS}

Chemicals and Media RPMI 1640 was obtained from Nissui Pharmaceutical Co. (Tokyo, Japan). Fetal calf serum (FCS) and trypsin solution were purchased from Gibco (Grand Island, NY). Pronase E and type I collagenase were purchased from Kaken Pharmaceutical Co. (Kyoto, Japan) and Nitta Gelatin Co. (Osaka, Japan), respectively. Sodium$N$-lauroylsarcosinate was from Wako Co. (Tokyo). Proteinase K, RNase A, Hoechst 33342 (Ho 342), 3-(4,5-dimethyl-thiazol-2-yl)-2,5-diphenyl tetrazolium bromide (MTT), and propidium iodide (PI) were from Sigma Co. (Tokyo). An enhanced chemiluminescence Western blotting detection kit was purchased from Amersham (Tokyo). Rabbit polyclonal antibody against HSP72 was kindly donated by Dr. H. Itoh (University of Akita, Akita, Japan). GGA and $\alpha$-tocopherol were generously provided by Eisai Co. (Tokyo).

The experiments and procedures were approved by the Animal Care Committee of Okayama University.

Preparation and Culture of Gastric Mucosal Cells Male guinea pigs (4 weeks of age) were purchased from Shimizu Co. (Kyoto). Gastric mucosal cells were isolated from guinea pig fundic glands, as described previously. ${ }^{6-9)}$ 
Isolated gastric mucosal cells $\left(1 \times 10^{6}\right.$ cells/dish $)$ were cultured for $48 \mathrm{~h}$ in RPMI 1640 containing $0.3 \% \mathrm{FCS}, 100 \mathrm{U} / \mathrm{ml}$ penicillin, and $100 \mu \mathrm{g} / \mathrm{ml}$ streptomycin in type-I collagencoated plastic culture plates (Iwaki) under the conditions of $5 \% \mathrm{CO}_{2} / 95 \%$ air and $37^{\circ} \mathrm{C}$. After removing non-adherent cells by washing with RPMI 1640, cells that were attached to the plate at about $50 \%$ confluence were used. Guinea pig gastric mucosal cell preparations under these conditions have been previously characterized, with the majority (about 90\%) of cells being identified as pit cells. ${ }^{\text {) }}$

Treatment of Cells with Gastric Stressors Cells were exposed to individual gastric stressors (ethanol, hydrogen peroxide or hydrochloric acid) by changing the entire bathing medium. For some experiments, cells were pre-incubated with $10^{-6} \mathrm{M}$ GGA or $3 \%$ ethanol for $2 \mathrm{~h}$. After removing these chemicals, cells were further incubated with one of the gastric stressors as described above.

Cell Viability Assay Cell viability was examined using the MTT assay. After exposure to one of the gastric stressors, cells were incubated with MTT $(1 \mathrm{mg} / \mathrm{ml})$ in PBS for $2 \mathrm{~h}$. Isopropanol and hydrochloric acid were added to the medium at final concentrations of $50 \%$ and $20 \mathrm{~mm}$, respectively. The optical density of the solution at $570 \mathrm{~nm}$ was determined using a spectrophotometer with a reference wavelength of $630 \mathrm{~nm}^{10}$

DNA Fragmentation Assay Apoptotic DNA fragmentation was monitored as previously described. ${ }^{11,12)}$ Briefly, cells were collected with a rubber policeman and suspended in $20 \mu \mathrm{l}$ of lysis buffer, consisting of $50 \mathrm{~mm}$ Tris- $\mathrm{HCl}$ ( $\mathrm{pH} \mathrm{7.8),}$ $10 \mathrm{~mm}$ EDTA, and $0.5 \%$ sodium- $N$-lauroylsarcosinate. Pro- teinase $\mathrm{K}$ was added to give a final concentration of $1 \mathrm{mg} / \mathrm{ml}$, and the lysate was incubated at $50^{\circ} \mathrm{C}$ for $2 \mathrm{~h}$. RNase $\mathrm{A}$ was then added to give a final concentration of $0.5 \mathrm{mg} / \mathrm{ml}$ and the solution was incubated again at $50{ }^{\circ} \mathrm{C}$ for a further $30 \mathrm{~min}$. Samples were analyzed by $2 \%$ agarose gel electrophoresis in the presence of $0.5 \mu \mathrm{g} / \mathrm{ml}$ ethidium bromide.

Nuclear Staining Assay for Necrosis For determination of the extent of necrosis, cells were washed with PBS and incubated with $0.17 \mathrm{~mm}$ Ho 342 and $100 \mu \mathrm{g} / \mathrm{ml}$ PI for $20 \mathrm{~min}$, following which they were analyzed using fluorescence microscopy as previously described. ${ }^{13}$

Immuno-blotting Analysis for HSP72 Cells were collected by centrifugation and dissolved with sodium dodecyl sulfate (SDS) sample buffer. After SDS polyacrylamide gel $(7.5 \%)$ electrophoresis, proteins were transferred to a polyvinylidene difluoride (PVDF) membrane (Nihon Millipore Ltd., Tokyo). The HSP72 band was detected using an antibody against the HSP72 ${ }^{14}$ ) and an enhanced chemiluminescence detection kit. The density of the band was determined by densitometric scanning using the National Institutes of Health (NIH) image programs (version 1.52). The relative density of the stained band was calculated using the formula: Relative density $(\%)=[$ density $($ treated $) /$ density (non-treated control) $] \times 100$.

Statistical Analysis All values are expressed as the mean \pm standard error (S.E.M.). A Student's $t$-test for paired results was performed for the evaluation of differences between the groups. Differences were considered to be significant for values of $p<0.05$
A
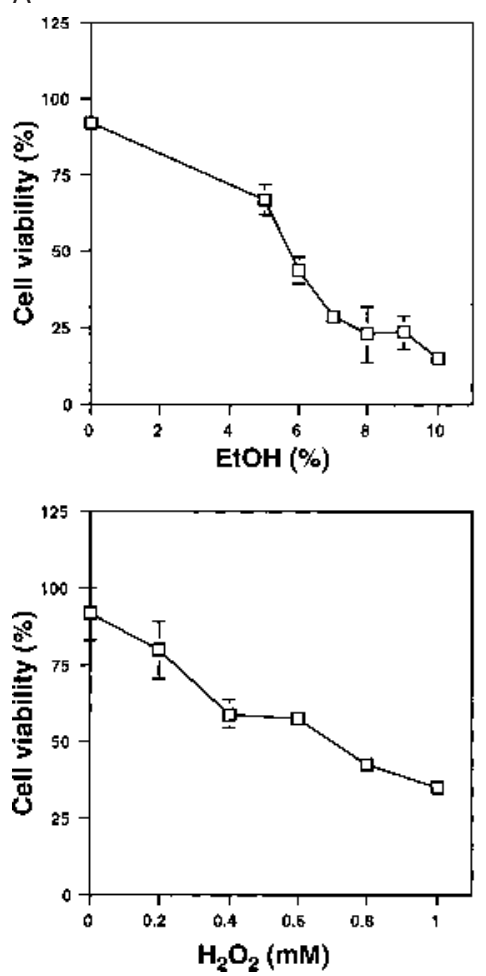

B

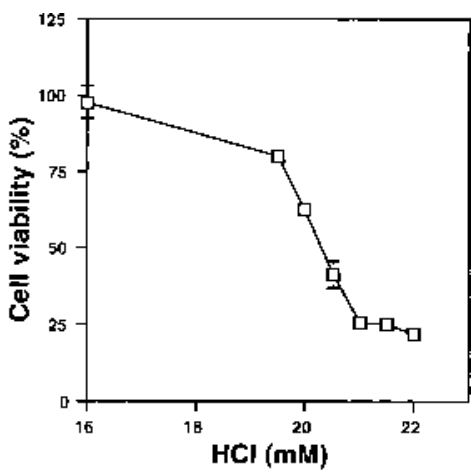

Incubation periods (h)

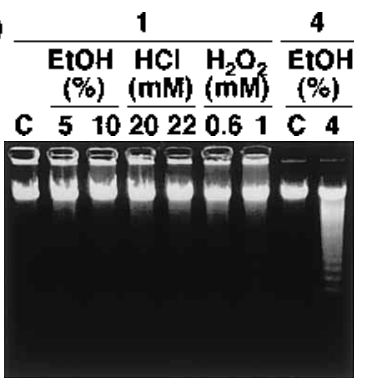

Fig. 1. Effects of Gastric Stressors on Cell Viability and Chromosomal DNA

Cultured gastric mucosal cells were incubated with indicated concentrations of ethanol (EtOH), hydrogen peroxide $\left(\mathrm{H}_{2} \mathrm{O}_{2}\right)$, or hydrochloric acid ( $\left.\mathrm{HCl}\right)$ for $1 \mathrm{~h}$. Control cells (indicated as C) were incubated for indicated periods in the absence of any form of gastric stressor. Cell viability was determined by MTT assay. Values are mean \pm S.D., $n=4$. (A) Chromosomal DNA was extracted and analyzed by $2 \%$ agarose gel electrophoresis. For positive control experiments of apoptosis, cells were incubated with $4 \%$ ethanol for $4 \mathrm{~h}$. (B) 


\section{RESULTS}

Effects of Gastric Stressors on Cell Viability and Chromosomal DNA We recently found that NSAIDs induced necrosis in guinea pig gastric mucosal cells in primary culture when the cells were treated for a short period of time $(1 \mathrm{~h})$, whereas the NSAIDs induced apoptosis when the cells were treated for a longer period of time $(16 \mathrm{~h})$ (Tomisato et al., submitted). We also found that various gastric stressors (ethanol, hydrogen peroxide and hydrochloric acid) induced apoptosis in guinea pig gastric mucosal cells in primary culture following exposure of the cells to the individual compounds for 4 or $8 \mathrm{~h}$ (Tsutsumi et al., submitted). In order to test the hypothesis that various gastric stressors generally could induce necrosis in guinea pig gastric mucosal cells in primary culture upon short-term treatment, we examined in the present study the mechanisms of cell death brought about by short-term $(1 \mathrm{~h})$ treatment of cells with the gastric stressors, ethanol, hydrogen peroxide, and hydrochloric acid.

We first examined the effects of these gastric stressors on cell viability; all of the gastric stressors decreased cell viability in a dose-dependent manner (Fig. 1A). The $\mathrm{IC}_{50}$ values of the cytotoxic effects of ethanol, hydrogen peroxide, and hydrochloric acid were about $6 \%, 20 \mathrm{~mm}$, and $0.6 \mathrm{~mm}$, respectively. In a previous study, when cells were treated with the gastric stressors for 4 or $8 \mathrm{~h}$, the $\mathrm{IC}_{50}$ values were much lower (Tsutsumi et al., submitted). Thus, the mechanism of cell death observed in Fig. 1A might be different from that seen for the long-term treatment. Since we found that these gastric stressors induced apoptosis under the conditions of the longterm treatment (Tsutsumi et al., submitted), the cell death observed for the experiments shown in Fig. 1A may be mediated by another form of cell death, i.e. necrosis.

Fragmentation of chromosomal DNA is generally seen with apoptosis but not with necrosis. ${ }^{15)}$ Thus, in order to understand the mechanism of cell death induced by these gastric stressors, we examined the state of chromosomal DNA following treatment of the cells for $1 \mathrm{~h}$ under the conditions used. As shown in Fig. 1B, none of the gastric stressors tested here induced apoptotic DNA fragmentation, even when they decreased cell viability to about $25 \%$ of control levels (Fig. 1A). When cells were treated with ethanol for longer periods, clear apoptotic DNA fragmentation was observed (Fig. 1B). The pattern of DNA fragmentation (i.e., the small molecular sizes of the fragments) was typical of that of apoptotic DNA fragmentation, based on the results from electrophoresis with the DNA ladder marker (data not shown). These results suggest that the gastric stressors employed induced necrosis in gastric mucosal cells when the cells were exposed to the stressors for just $1 \mathrm{~h}$.

Effects of Gastric Stressors on Membrane Integrity and Chromatin Structure Since some forms of apoptosis are not accompanied by DNA fragmentation, we could not simply conclude, based only on the data shown in Fig. 1, that cell death by these gastric stressors was mediated by necrosis. We therefore carried out double-staining experiments with PI and Ho 342 to further elucidate the mechanism of cell death. Because necrotic cells lose their membrane integrity, PI staining causes pink nuclear staining in necrotic cells, whereas living cells and apoptotic cells do not stain with PI, because they are able to pump PI out of the cell. ${ }^{13)}$

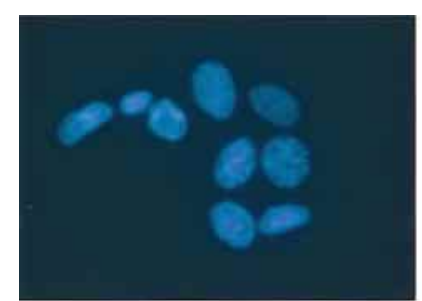

Control $1 \mathrm{~h}$

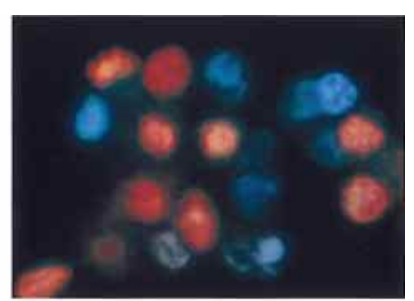

$10 \%$ EtOH $1 \mathrm{~h}$

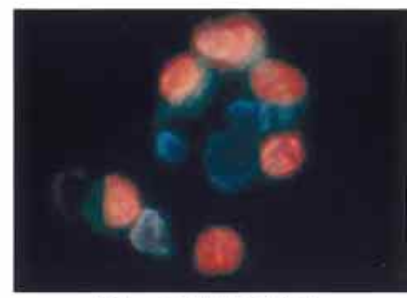

$22 \mathrm{mM} \mathrm{HCl} 1 \mathrm{~h}$

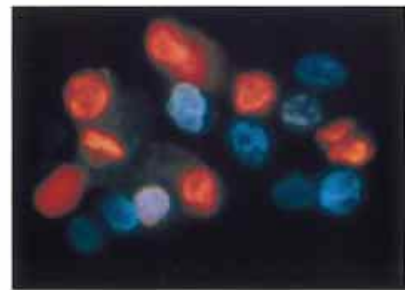

$1 \mathrm{mM} \mathrm{H}_{2} \mathrm{O}_{2} 1 \mathrm{~h}$

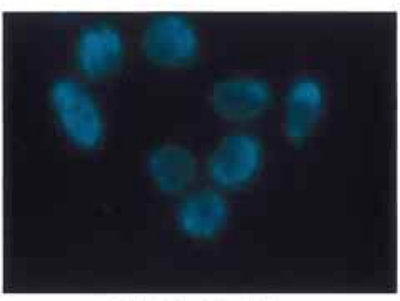

Control $4 \mathrm{~h}$

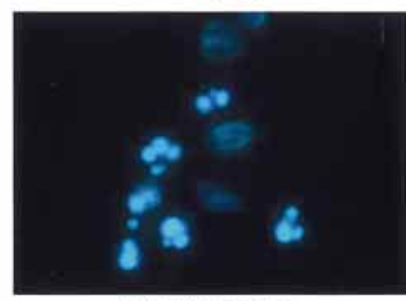

$4 \%$ EtOH $4 \mathrm{~h}$
Fig. 2. Effects of Gastric Stressors on Membrane Integrity and Chromatin Structure

Cultured gastric mucosal cells were incubated with indicated concentrations of gastric stressors for $1 \mathrm{~h}$. After staining with both Ho 342 and PI, cells were observed under fluorescence microscope. For positive control experiments of apoptosis, cells were incubated with $4 \%$ ethanol for $4 \mathrm{~h}$.

Ho 342 can be used to help visualize condensed chromatin, which is generally observed with apoptosis. ${ }^{13)}$ For these experiments, we used concentrations of gastric stressors capable of bringing about a $75 \%$ decrease in cell viability (ethanol, 10\%; hydrochloric acid, $22 \mathrm{~mm}$; hydrogen peroxide, $1 \mathrm{~mm}$ ). Control cells did not stain with PI and did not show chromatin condensation (Fig. 2). In contrast, cells treated with any one of the gastric stressors for $1 \mathrm{~h}$ showed pink nuclear staining without chromatin condensation (Fig. 2). These results strongly suggest that the short-term treatment of cells with the gastric stressors employed here causes cell death through necrosis. Cells treated with $4 \%$ ethanol for $4 \mathrm{~h}$ were not stained with PI but did show chromatin condensation (Fig. 2), suggesting that apoptosis was induced under these conditions, as has been described previously. ${ }^{16)} \mathrm{Be}-$ cause cells were not fixed in these experiments, they were only weakly stained with Ho 342 .

Effect of GGA and Low Concentrations of Ethanol on Necrosis Due to Various Gastric Stressors Pre-induction of HSPs is thought to make cells resistant to subsequent 

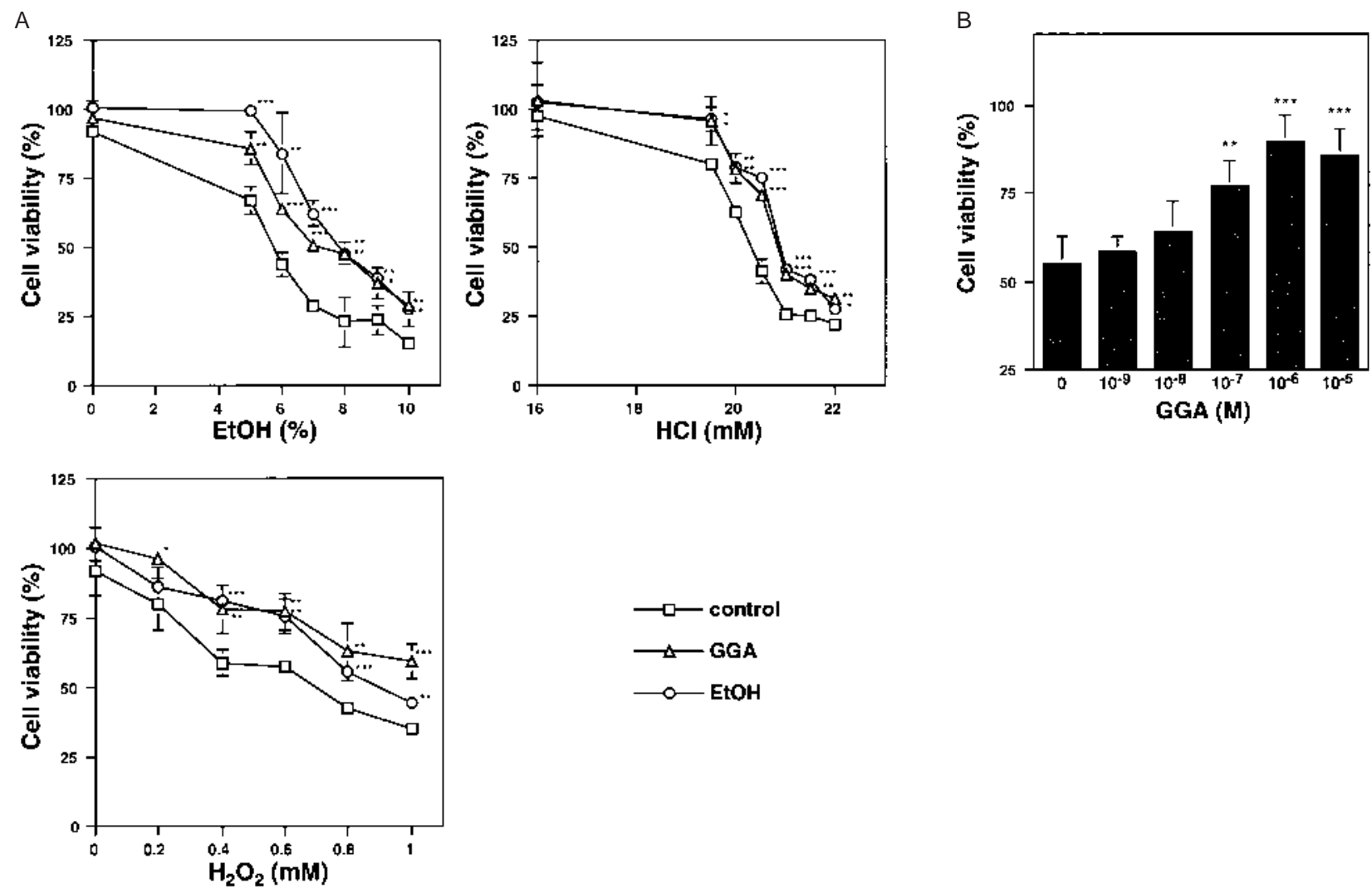

Fig. 3. Effect of GGA and Low Concentrations of Ethanol on Necrotic Cell Death Following Short-Term Treatment with Gastric Stressors

Gastric mucosal cells were pre-incubated with GGA $\left(10^{-6} \mathrm{M}\right.$ (A) or indicated concentrations (B)) or $3 \%$ ethanol for $2 \mathrm{~h}$. After washing out these compounds, cells were further incubated with indicated concentrations of gastric stressors (A) or $6 \%$ ethanol (B) for $1 \mathrm{~h}$. Cell viability was determined by MTT assay. Values are mean \pm S.D., $n=4 * * *, p<0.001$; **, $p<0.01 ; *, p<0.05$ by Student's $t$-test compared with not pre-treated and GGA or ethanol-pre-treated cells.

stressor-treatment. ${ }^{5)}$ Using gastric mucosal cells in primary culture, we previously showed that pre-induction of HSPs makes cells resistant to necrotic cell death induced by NSAIDs. ${ }^{7)}$ Pre-induction of HSPs also makes cells resistant to cell death induced by ethanol. $\left.{ }^{6}\right)$ We therefore examined here the effect of pre-induction of HSPs on necrotic cell death due to these gastric stressors, using GGA, an anti-ulcer drug with the capability of inducing HSP-expression. ${ }^{6}$ ) When cells were pre-treated with $10^{-6} \mathrm{M}$ GGA for $2 \mathrm{~h}$, the viability of cells subsequently exposed for $1 \mathrm{~h}$ to each dosage of the gastric stressors used (ethanol, hydrogen peroxide, and hydrochloric acid) significantly increased (Fig. 3A). A dose-response experiment revealed that more than $10^{-7} \mathrm{M}$ GGA was required for increase in cell viability in the presence of ethanol (Fig. 3B). GGA was dissolved in ethanol containing $0.25 \% \alpha$-tocopherol, and we confirmed that these compounds, which were used at final concentrations of 0.00025 and $0.1 \%$, respectively, did not alter the cytotoxic effect of the gastric stressors used (data not shown).

Although GGA is known to affect cell physiology in ways other than the induction of HSP-expression. ${ }^{17-20)}$ we consider that the HSPs induced by GGA were responsible for its cytoprotective effect against the various gastric stressors (Fig. 3). To support this conclusion, we first set out to confirm, using immuno-blotting techniques, that GGA induces HSP expression under the conditions used. As shown in Fig. 4, GGA induced HSP72 expression, as has been described previously, ${ }^{6}$ although we did not find such a strong effect. This was probably due to the presence of serum in the
A
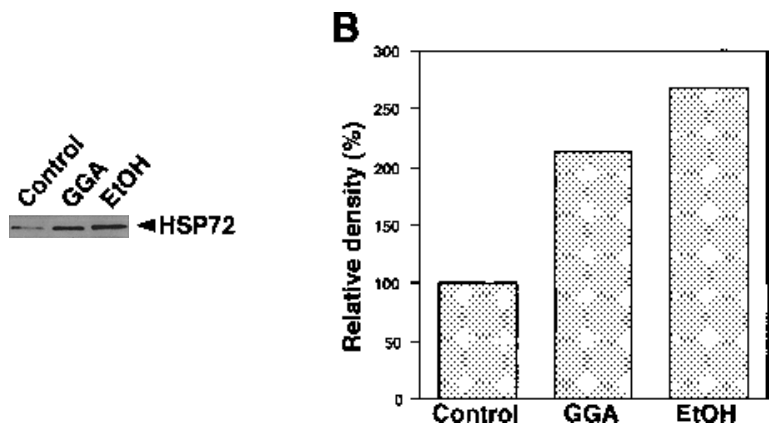

Fig. 4. Induction of HSP72 Expression by GGA or a Low Concentration of Ethanol

Gastric mucosal cells were incubated with $10^{-6} \mathrm{M}$ GGA or $3 \%$ ethanol for $2 \mathrm{~h}$. HSP72 was detected by immuno-blotting (A) and determined by densitometric scanning (B). Similar results were obtained in three separate experiments.

medium because removal of the serum caused a higher apparent HSP72 expression (data not shown). Similar results were obtained for HSP27 (data not shown).

We next tested whether or not the induction of HSP expression by means other than GGA protects gastric mucosal cells from stressor-induced necrotic cell death. We found that treatment of cells with $3 \%$ ethanol induced HSP72 expression to a similar extent as that obtained with GGA (Fig. 4). As shown in Fig. 3, compared to non-treated cells exposed to gastric stressors, pre-treatment of gastric mucosal cells with $3 \%$ ethanol made cells significantly more resistant to subsequent treatment with each of the gastric stressors employed 
in this study. These results support the notion that pre-induction of HSPs protects gastric mucosal cells from necrosis caused by various gastric stressors.

\section{DISCUSSION}

In this report, we clearly showed that short-term treatment of gastric mucosal cells in primary culture with each of the gastric stressors tested here (ethanol, hydrogen peroxide, and hydrochloric acid) induced necrosis. These conclusions are made on the basis of results obtained from experiments in which we examined cell viability, DNA fragmentation, chromatin condensation, and membrane permeability (PI staining). Since the properties of gastric mucosal cells in primary culture are thought to closely mimic gastric mucosal cells in vivo, we consider that necrosis induced by these gastric stressors in vitro reproduces stressor-induced necrosis in gastric mucosa in vivo. This system may be useful in elucidating mechanisms to protect gastric mucosal cells from necrotic cell death, which is related to gastric ulcers and gastritis.

In contrast to the results of short-term treatment of cells with gastric stressors as shown in this paper, we recently found that the long-term treatment of gastric mucosal cells with each of these gastric stressors induced apoptosis at concentrations lower than those required for necrosis (Tsutsumi et al., submitted). In relation to NSAIDs, we demonstrated that NSAIDs induce necrosis when cells were treated for a short period of time at a high concentration, whereas NSAIDs induced apoptosis when cells were treated for a long period of time at the lower concentration (Tomisato et al., submitted). All these data allow us to propose that various gastric stressors generally induce necrosis and apoptosis in cultured gastric mucosal cells depending on the conditions employed. Such cell death may decrease the number of functional gastric mucosal cells and causes gastric ulcers and gastritis in vivo. Thus, in order to protect gastric mucosa from various gastric stressors, suitable drugs are necessary that can inhibit both necrosis and apoptosis induced by any of these gastric stressors. We consider that non-toxic compounds that can induce HSPs, such as GGA, may be good candidates for such a drug. We showed here that GGA and low concentrations of ethanol, both of which induced HSPs, protected gastric mucosal cells from necrotic cell death due to ethanol, hydrogen peroxide, or hydrochloric acid. We have also shown previously that GGA and low concentrations of ethanol also protected gastric mucosal cells from necrotic cell death induced by NSAIDs. ${ }^{7}$ Furthermore, these HSP-inducers inhibited gastric stressor-induced apoptosis in gastric mucosal cells in primary culture. ${ }^{16)}$ Thus, it seems that preinduction of HSPs in gastric mucosal cells protects the cells from both necrotic and apoptotic cell death due to all of gastric stressors. Identification of HSP, which is involved in protection of cells from apoptotic and necrotic cell death, seems to be important.

Acknowledgements We thank Dr. H. Itoh (University of Akita) for kindly providing us with antibody against HSP72. This work was supported by Grants-in-Aid for Scientific Research from the Ministry of Education, Science, Sports, Culture, and Technology of Japan.

\section{REFERENCES}

1) Buttke T. M., Sandstrom P. A., Immunol. Today, 15, 7-10 (1994).

2) Cosulich S., Clarke P., Curr. Biol., 6, 1586-1588 (1996).

3) Szabo I., Tarnawski A. S., J. Physiol. Pharmacol., 51, 3-15 (2000).

4) Smoot D. T., Gastroenterology, 113, S31-34 (1997).

5) Tsukimi Y., Okabe S., Biol. Pharm. Bull., 24, 1-9 (2001).

6) Hirakawa T., Rokutan K., Nikawa T., Kishi K., Gastroenterology, 111, 345-357 (1996).

7) Tomisato W., Takahashi N., Komoto C., Rokutan K., Tsuchiya T., Mizushima T., Dig. Dis. Sci., 45, 1674-1679 (2000).

8) Nakamura K., Rokutan K., Marui N., Aoike A., Kawai K., Gastroenterology, 101, 161-166 (1991).

9) Teshima S., Kutsumi H., Kawahara T., Kishi K., Rokutan K., Am. J. Physiol. Gastrointest. Liver Physiol., 279, G1169-G1176 (2000).

10) Yamamura Y., Noda M., Ikawa Y., Proc. Natl. Acad. Sci. U.S.A., 91, 8866 -8870 (1994).

11) Tsutsumi S., Rokutan K., Tsuchiya T., Mizushima T., Biol. Pharm. Bull., 22, 886-887 (1999).

12) Tsutsumi S., Rokutan K., Tsuchiya T., Mizushima T., Dig. Dis. Sci., 45, 291-297 (2000).

13) Herceg Z., Wang Z. Q., Mol. Cell. Biol., 19, 5124-5133 (1999).

14) Wakui H., Itoh H., Tashima Y., Kobayashi R., Nakamoto Y., Miura A. B., Int. J. Biochem., 23, 975-978 (1991).

15) Gerschenson L. E., Rotello R. J., FASEB J., 6, 2450-2455 (1992).

16) Mizushima T., Tsutsumi S., Rokutan K., Tsuchiya T., Dig. Dis. Sci., 44, 510-514 (1999).

17) Terano A., Hiraishi H., Ota S., Sugimoto T., Digestion, 33, 206-210 (1986).

18) Namiki T., Egawa M., Inoue S., Tominaga S., Takamura Y., Am. J. Physiol., 266, R503-509 (1994).

19) Hassan S., Kinoshita Y., Okada A., Maekawa T., Fukui H., Chiba T., J. Gastroenterol. Hepatol., 13, 809-815 (1998).

20) Rokutan K., Teshima S., Kawai T., Kawahara T., Kusumoto K., Mizushima T., Kishi K., J. Gastroenterol., 35, 673-681 (2000). 